\begin{tabular}{|l|l|l|l|l|}
\hline Cuadernos I. Geográfica & $20-21$ & pp. 85-98 & Logroño & 1994-95 \\
\hline
\end{tabular}

\title{
REVEGETACIÓN NATURAL EN TALUDES DE PISTAS FORESTALES (Sistema Ibérico, La Rioja, España)
}

\author{
V. LARREA SÁENZ ${ }^{1}$ \\ J. ARNÁEZ VADHLLO ${ }^{2}$ \\ L. ORTIGOSA IZQUIERDO'
}

RESUMEN: En este trabajo se describen la dinámica y organización espacial de la revegetación natural en taludes de pistas forestales. Los autores constatan la existencia de diferencias significativas en el cubrimiento vegetal del corte-talud superior (T1) y talud inferior de las pistas (T2), y establecen las estrategias de recolonización. La altitud, la pendiente de los taludes y los procesos de erosión se han mostrado muy influyentes en la evolución de la recolonización vegetal.

ABSTRACT: In this paper the authors describe the dynamic and the spatial organization of the natural revegetation on road-sides of hillroads. They show the existence of significant differences in the percentage of vegetal cover between the road-cuts (T1) and the debris of the first meters downslope of the road (T2), and they establish the recolonization strategies. Altitude, road-sides gradient, and erosion processes are very influential in the develop of the revegetation.

Palabras clave: Pistas forestales, revegetación natural, erosión, Sistema Ibérico.

Key words: Hill-roads, natural revegetation, erosion, Iberian System.

1. Departamento de Geografía. Instituto de Estudios Riojanos. Logroño

2. Area de Geografia Física (DCHS). Universidad de La Rioja. Logroño 
V. LARREA SÁENZ - J. ARNÁEZ VADILLO - L. ORTIGOSA IZQUIERDO

\section{Introducción}

El trazado de una pista forestal en una ladera de montaña supone, en el momento de su construcción, importantes alteraciones en el medio natural; al movilizarse cientos de metros cúbicos de suelo, roca y vegetación (FOLCH GUILLEN, 1977). Con posterioridad, en unos taludes desnudos, afectados por las escorrentías, se detectan diversos procesos de erosión que suelen ser controlados con la aplicación de técnicas de protección y un mantenimiento adecuado (ARNÁEZ VADILLO \& LARREA, 1991; ARNÁEZ VADILLO \& LARREA, 1995; LARREA \& ARNÁEZ VADILLO, 1996). Estas técnicas son variadas y dependen de los presupuestos disponibles y de los problemas que tratan de evitarse. Sin ánimo de ser exhaustivos, las acciones más habituales van destinadas a controlar la erosión de los taludes y a reconducir, en la medida de lo posible, la circulación del agua. Para el primer fin se instalan mallas metálicas, muros de hormigón, muros de piedra, revestimientos de cemento o terracetas, tratando de evitar, de este modo, la actividad de la meteorización física, las rigolas o los movimientos en masa (FOOKES et al., 1985). Para reconducir la escorrentía procedente de la ladera se diseñan drenajes en las cunetas, al pie del corte-talud, con el fin de evacuarla sin que llegue a afectar a la calzada de la pista.

La restauración de los taludes por medio de la plantación de arbustos o siembra de herbáceas también es utilizada en la lucha contra los procesos erosivos y en la disminución del impacto visual que representan las pistas en el paisaje. La cubierta vegetal favorece la intercepción de la precipitación y un mayor consumo de agua a través de la evapotranspiración. Pero, además, incrementa la resistencia del suelo por la sujección que ejercen las raíces. DISEKER \& RICHARDSON (1962) ponen de manifiesto, a partir de diversos trabajos experimentales, la efectividad de la vegetación como control de la erosión en taludes de pistas de montaña. En taludes de escombreras mineras, con problemática muy semejante a la de las pistas forestales, la restauración vegetal se ha mostrado determinante en la reducción de la producción anual de escorrentía (NICOLAU IBARRA, 1992).

En las pistas forestales estudiadas del Sistema Ibérico occidental (La Rioja) no se ha aplicado a los taludes ningún tratamiento de repoblación vegetal. No obstante, una vez construidas las pistas, los taludes, inicialmente desnudos, comienzan a ser colonizados con mayor o menor rapidez a partir de la vegetación existente en la ladera. El objetivo de este trabajo es explicar la organización espacial y dinámica de la vegetación en los taludes así como su relación con la actividad de los procesos erosivos. 
REVEGETACIÓN NATURAL EN TALUDES DE PISTAS FORESTALES

\section{Area de estudio}

El sector occidental del Sistema Ibérico, localizado en la mitad sur de La Rioja, está compuesto por un conjunto de macizos que, a pesar de su parecido aspecto, presentan diferencias significativas. En concreto, a medida que nos trasladamos de Oeste a Este, descienden las altitudes de las líneas de cumbres, de modo que frente a divisorias por encima de los $2000 \mathrm{~m}$ de la Sierra de la Demanda, Sierra de Urbión o Sierra de Cebollera se encuentran otras que apenas superan los $1400 \mathrm{~m}$ (Sierra de Alcarama, en el extremo oriental de La Rioja). Además, desde un punto de vista litológico, los materiales principales de la Demanda, macizo paleozoico rejuvenecido por el plegamiento alpino, son las cuarcitas y las pizarras. Por el contrario, en el resto de las sierras predominan las rocas cretácicas (cuarzoarenitas, calizas, areniscas y arcillas).

Con relación al clima, en el sector occidental las temperaturas son más bajas y las precipitaciones más abundantes que en el oriental. La Sierra de la Demanda muestra un clima con cierta tendencia oceánica, ya que las precipitaciones superan los $947 \mathrm{~mm}$ a los $1020 \mathrm{~m}$ (estación de Valvanera) y posiblemente los 1500 mm en las líneas de cumbres. Por otro lado, y si exceptuamos el verano, la distribución estacional de las lluvias es muy regular. En el alto valle del Iregua la precipitación recogida es de $636 \mathrm{~mm}$ a los $980 \mathrm{~m}$ (estación de Ortigosa) (NUÑEZ OLIVERA \& MARTÍNEZ ABAIGAR, 1991). La menor importancia de las lluvias invernales y el descenso de las temperaturas medias perfilan un clima de tendencia continental. Por último, al Este del Iregua pueden detectarse unas características más próximas a los climas mediterráneos, con temperaturas medias anuales en torno a los 11-12 y precipitaciones entre los $400-600 \mathrm{~mm}$.

La vegetación natural se adapta a estas condiciones climáticas. Por ello, al Oeste, en la Sierra de la Demanda, Urbión y Cebollera, los hayedos (Fagus silvatica) son los bosques más representativos en ambientes húmedos, por encima de los $1200 \mathrm{~m}$. Estos son sustituidos por los robledales (Quercus pyrenaica) en laderas más soleadas y de menor altitud. Al Este del Iregua, el hayedo sólo se localiza en las cabeceras de los barrancos, con orientación umbría. Otras especies, como el roble (Quercus pyrenaica), el quejigo (Quercus faginea) o la carrasca (Quercus rotundifolia), son mucho más representativas. No obstante, en Cameros Viejo y en la Sierra de Alcarama el matorral es el conjunto vegetal que ocupa una mayor superficie (Thymus vulgaris, Genista scorpius, en suelos básicos, y Cistus laurifolius, en suelos silíceos).

Las pistas forestales estudiadas recorren los ambientes descritos (Figura 1). En concreto, se han seleccionado seis pistas en los macizos más representativos (Sierra de la Demanda, Sierra de Cebollera, Cameros Nuevo, Cameros Viejo y Sierra de Alcarama) y una séptima en los relieves más elevados de la Depresión del Ebro (Cabimonteros). Para más detalles puede consultarse la tabla 1. 
V. LARREA SÁENZ - J. ARNÁEZ VADILLO - L. ORTIGOSA IZQUIERDO

Tabla 1. Características generales de las pistas seleccionadas

\begin{tabular}{|c|c|c|c|c|c|}
\hline Pista & Macizo & Litología & $\begin{array}{c}\text { Alt. max. } \\
(\mathrm{m})\end{array}$ & $\begin{array}{c}\text { Alt. min. } \\
(\mathrm{m})\end{array}$ & Fecha constr. \\
\hline P1 & S. Demanda & Pizarras & 1525 & 970 & antes de 1977 \\
P2 & S. Cebollera & Cuarzoarenitas & 1980 & 1200 & $1973-80$ \\
P3 & C. Nuevo & Calizas & 1430 & 980 & antes de 1977 \\
P4 & C. Viejo & Yesos/calizas & 1145 & 755 & $1985-88$ \\
P5 & C. Viejo & Cuarzoarenitas & 1245 & 750 & $1987-88$ \\
P6 & S. Alcarama & Arcillas & 1220 & 740 & $1980-84$ \\
P7 & Cabimonteros & Conglomerados & 1320 & 770 & $1987-88$ \\
\hline
\end{tabular}

P1 (Valgañón-Ezcaray), P2 (Hoyos de Iregua), P3 (Nieva-Gamellones), P4 (Zenzano), P5 (Sta. Marina), P6 (S. Alcarama), P7 (Ocón-Arnedillo).

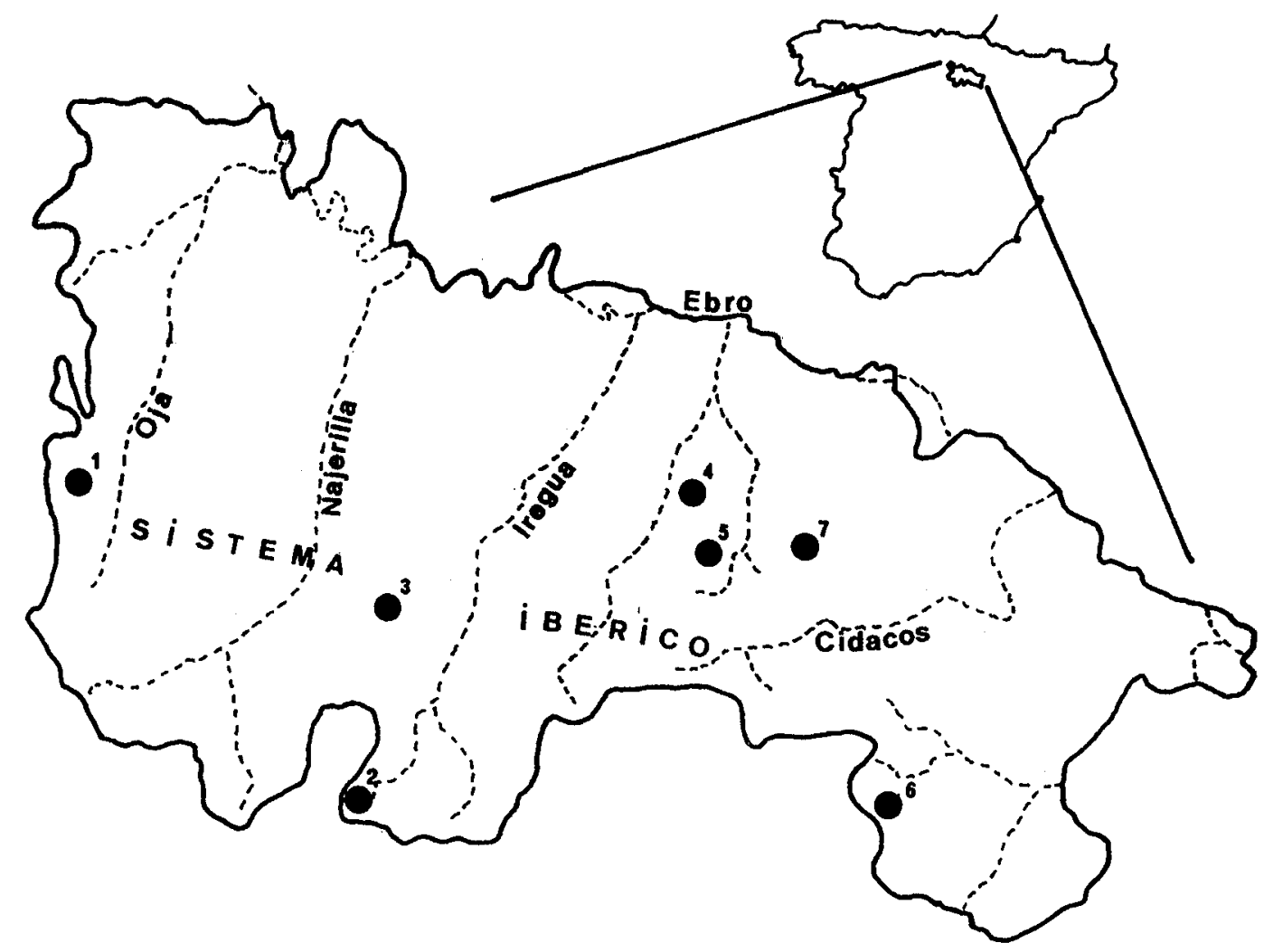

Figura 1. Localización del área de estudio. P1 (Valgañón-Ezcaray, Sierra de la Demanda), P2 (Hoyos de Iregua, Sierra de Cebollera), P3 (Nieva-Gamellones, Cameros Nuevo), P4 (Zenzano, Cameros Viejo), P5 (Sta. Marina, Cameros Viejo), P6 (Sierra de Alcarama), P7 (Ocón-Arnedillo, Cabimonteros). 
REVEGETACIÓN NATURAL EN TALUDES DE PISTAS FORESTALES

\section{Métodos}

Para la realización del estudio, una vez elegidas las pistas forestales, se seleccionaron, a partir de la fotografía aérea, 59 transectos de $20 \mathrm{~m}$. El número de transectos por pista fue proporcional a la longitud de la pista, intentando que estuviesen distanciados entre $1500-2000 \mathrm{~m}$. En cada transecto se diferenció el corte-talud superior (T1), que se configura al insertar la pista en la ladera, y el talud inferior (T2), o sector inmediatamente aguas abajo de la calzada donde se ha acumulado el material de desmonte.

Se pudo disponer, pues, de un total de 118 fichas con información que incluía datos de las características morfométricas de los taludes, porcentaje de cubrimiento vegetal, especies más representativas y formas-procesos de erosión.

Por lo que respecta a la vegetación, el muestreo se realizó utilizando una retícula de $0.25 \mathrm{~m}^{2}$, situándola a 2,11 y $18 \mathrm{~m}$ del punto inicial del transecto y a distintos niveles del talud (superior, medio y pie de talud). Con el fin de facilitar en este trabajo el manejo de la información y su análisis estadístico, las especies vegetales fueron agrupadas en tres categorías:

a) Vegetación arbórea. Las especies más representativas dentro de este grupo son: el haya (Fagus silvatica), el pino silvestre (Pinus silvestris), el roble (Quercus pyrenaica) y el quejigo (Quercus faginea).

b) Matorral. Las especies que se incluyen en esta categoría son: brezos (Erica australis ssp. aragonensis, Erica arborea, Erica cinerea, Erica vagans), brecina (Calluna vulgaris), enebro (Juniperus nana), piorno (Cytisus purgans), arándano (Vaccinium myrtillus), escoba (Genista florida), retama (Cytisus scoparius), jara (Cistus laurifolius), cantueso (Lavandula stoechas), tomillos (Thymus vulgaris, Thymus mastichina, Thymus zygis), aulagas (Genista bispanica, Gesnita scorpius), espliego (Lavandula latifolia), romero (Rosmarinus officinalis), boj (Buxus sempervirens), endrino (Prunus spinosa), rosales (Rosa canina, Rosa pouzinii), majuelo (Crataegus monogyna), zarzamora (Rubus fruticosus).

c) Vegetación herbácea: digital (Digitalis purpurea), fresa silvestre (Fragaria vesca), gordolobos (Verbascum pulverulentum, Verbascum thapsus), gramíneas (Deschampsia flexuosa, Lolium perenne, etc.), chirivita (Bellis perennis), cardos (Silybum marianum, Onopordum acantbium, Cirsium arvense), centaureas (Centaurea calcitrapa, Centaurea niga, Centaurea jacea), pulicaria (Pulicaria dysenterica), cerraja común (Sonchus asper), ortiga (Labium purpureum), viborera (Echium vulgare), bugloso (Anchusa officinalis), menta (Mentba sp.), tréboles (Trifolium pratense, Trifolium repens), nazareno (Muscari comdsum), Silene vulgaris, achicoria (Cichorium intybus), mielenrama (Acbilea millefoium), carlina de monte (Carlina vulgaris), llantén 
menor (Plantago lanceolata), correhuelas (Calistegia sepium, Convulvus arvensis), alcaravea (Carum carvim), matacandil (Sisybrium irio), rabo de gato (Sideritis birsuta), abrotano hembra (Santolina chamaecyparissus), gatuna (Ononis spinosa), siempreviva (Helicbrysum stoechas), helecho (Pteridium aquilinum).

A pesar de la variedad de microambientes geomorfológicos detectados en los taludes de pistas forestales (ARNÁEZ VADILLO \& LARREA, 1991; LARREA \& ARNÁEZ VADILLO, 1996), para este trabajo sólo se consideró aquellos procesos indicativos de un grado de erosión severo: arroyamiento difuso, incisiones y deslizamientos (ARNÁEZ VADILLO \& LARREA, 1994). Se cuantificó la superficie que ocupaban en cada uno de los transectos para posteriormente, y a través de un cluster, establecer modelos erosivos que relacionamos con el grado de revegetación de los taludes.

\section{Resultados y discusión}

En el conjunto de las pistas estudiadas el porcentaje medio de cubrimiento vegetal de los taludes es de un $40.38 \%$. Por tipos de vegetación es el matorral el que ocupa una mayor superficie, con un $14.41 \%$. En segundo lugar, destaca la cubierta herbácea (14\%) y, finalmente, la arbórea (11.97\%). Estos valores se ven modificados, tanto por lo que respecta al grado de cubrimiento como a los grupos de vegetación, si diferenciamos la dinámica de la revegetación en el talud superior (T1) e inferior (T2) y en los distintos sectores de ambos (ver Fig.2).

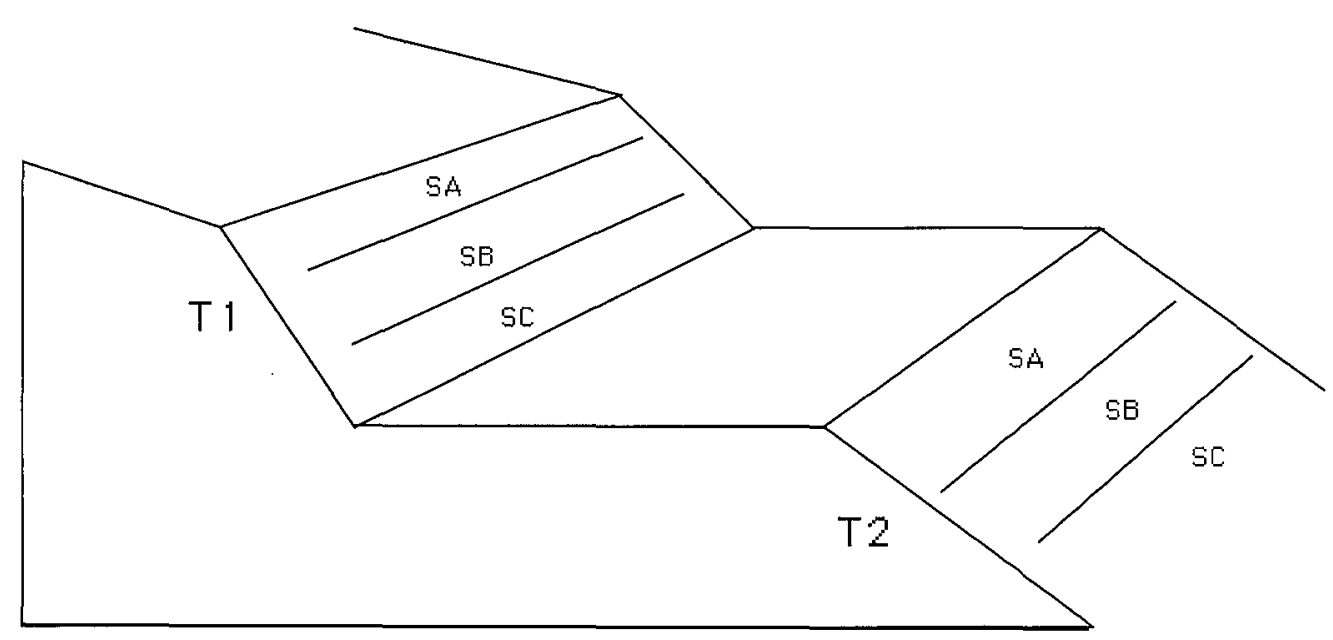

Figura 2. Secciones consideradas en los taludes de pistas forestales. T1: corte-talud superior; T2: talud inferior (derrubios procedentes del desmonte de la ladera); SA: sector superior del talud; SB: sector medio del talud; SC: pie del talud. 
REVEGETACIÓN NATURAL EN TALUDES DE PISTAS FORESTALES

Tabla 2. Cubrimiento vegetal en los taludes de las pistas forestales (\%)

\begin{tabular}{|c|c|c|c|c|}
\hline Talud & c. arbórea & matorral & c. herbácea & total \\
\hline T1 & 4.0 & 7.5 & 10.2 & 21.8 \\
T2 & 19.9 & 21.3 & 17.7 & 59.0 \\
\hline
\end{tabular}

A partir de la Tabla 2 se puede señalar que en el talud superior la vegetación ha recolonizado sólo el $21.8 \%$ de su superficie, apreciándose un mayor dinamismo del matorral y las herbáceas $(7.5 \%$ y $10.2 \%$, respectivamente). Por el contrario, en el talud inferior la revegetación alcanza el $59 \%$, destacando la importancia que adquiere la cubierta arbórea.

Estos resultados ponen de manifiesto la adaptación de las distintas especies vegetales a las características de ambos taludes. El talud superior (T1) presenta una pendiente más inclinada y una mayor actividad, como veremos más adelante, de los movimientos en masa (deslizamientos) que dificultan el enraizamiento de las plantas. En concreto, y por lo que respecta al primer aspecto, la inclinación media de estos taludes es de $43.5^{\circ}$ mientras que la superficie media afectada por deslizamientos ha sido evaluada en un 16.5\%. En el talud inferior (T2) la reintroducción de la vegetación resulta más fácil, si tenemos en cuenta la menor pendiente $\left(34^{\circ}\right)$ y las características del material que conforman estos sectores: derrubios sueltos, acompañados de una matriz fina, que facilitan el agarre de las plantas. Incluso no hay que olvidar que este material proviene de la sección de la ladera que hubo que remover para la apertura de la pista forestal. No es extraño, pues, que en él hayan permanecido semillas que han brotado posteriormente. Tampoco es inhabitual en laderas cubiertas de bosque que el material de desmonte sólo haya enterrado al arbolado parcialmente (primeros centímetros o metros del tronco). Estos enraizados al suelo de la ladera siguen su crecimiento, pudiendo favorecer el desarrollo de un pequeño sotobosque en el talud.

Si estudiamos la distribución de la vegetación dentro del propio talud, distinguiendo tres sectores (superior, medio y pie de talud) (ver Fig. 2), se comprueba que en $\mathrm{T} 1$ el sector superior (SA) manifiesta los porcentajes de cubrimiento vegetal más elevados (30.2\%), seguido por el pie del talud (SC): $21.3 \%$. Por el contrario, el sector medio del talud (SB) se encuentra mucho más desnudo, ya que sólo logra un cubrimiento del $13.4 \%$ (Fig. 3). Esta distribución se mantiene tanto para la vegetación arbórea como para el matorral. Los datos ponen de manifiesto las mejores condiciones del sector superior, húmedo, ya que a él van a parar las escorrentías subsuperficiales que circulan por los 
primeros centímetros del suelo, y próximo a las especies vegetales que cubren la ladera de las que partirá el proceso de recolonización. El pie del talud se beneficia del material proveniente de las partes altas del talud, en muchos casos bloques de suelo que descienden acompañados de vegetación, semillas $y$ nutrientes. También el agua tiende a acumularse en estos sectores, sobre todo si no existen adecuados sistemas de drenaje.

TALUD SUPERIOR (T1)
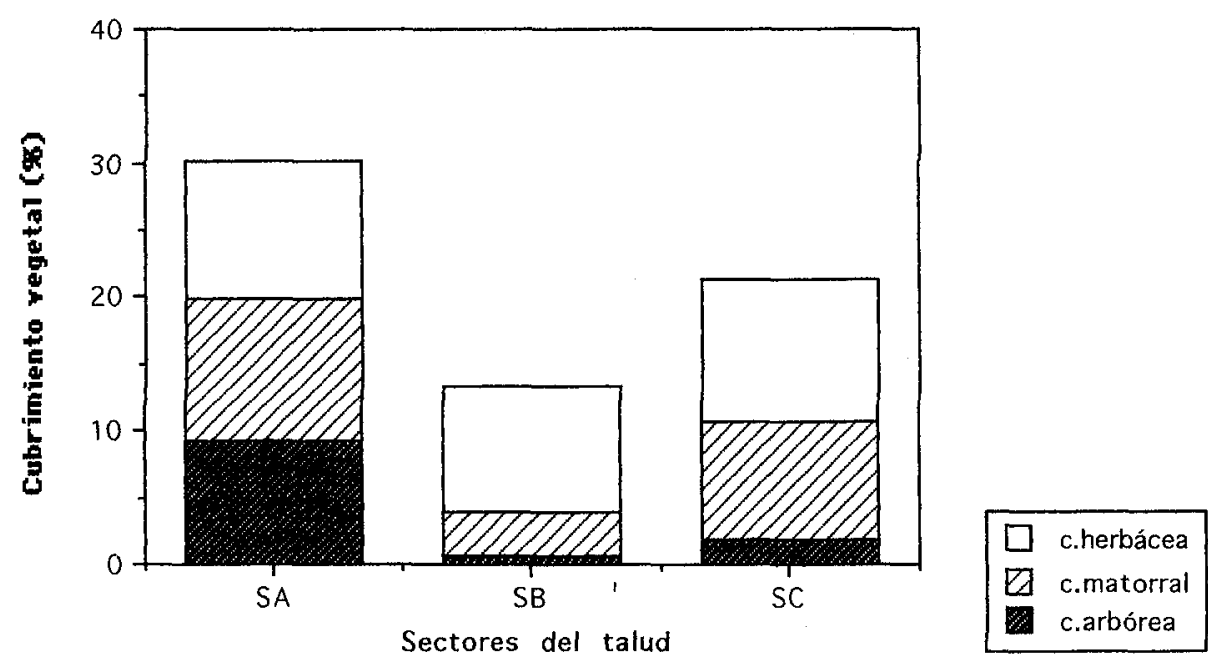

TALUD INFERIOR (T2)

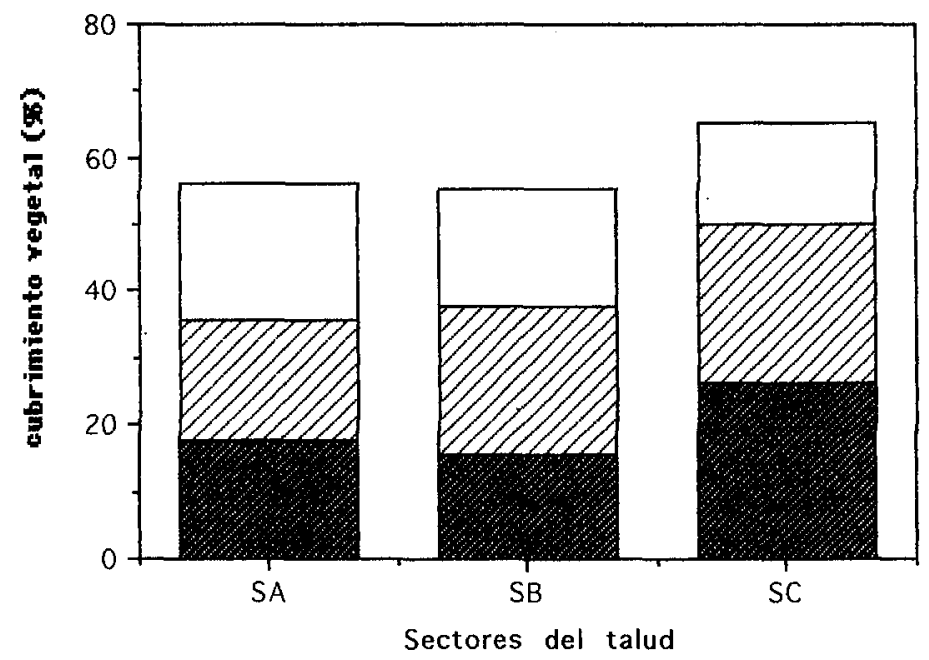

Figura 3. Cubrimiento vegetal en los distintos sectores de los taludes de las pistas forestales (SA: sector superior del talud; SB: sector medio del talud; SC: pie del talud). 
En el talud inferior (T2) los mayores porcentajes de cubrimiento vegetal se encuentran en el pie del talud (SC), con un 65.2\%. Aquí la cubierta arbórea es muy superior a los otros tipos de vegetación. En el sector superior (SA) y medio (SB) el cubrimiento alcanza el $56.3 \%$ y $55.2 \%$, respectivamente. Como en el caso anterior, puede entreverse una cierta relación entre el sector con mayor porcentaje de vegetación y la proximidad a los núcleos a partir de los cuales comienza el proceso de revegetación: en este caso, la ladera aguas abajo de la pista. No obstante, en T2, y por lo que respecta a la cubierta arbórea, se dispone no sólo de árboles jóvenes consecuencia de los procesos de recolonización, sino también de otros que no han podido ser cubiertos totalmente por el material de desmonte.

La dinámica de la revegetación en los taludes de las pistas estudiadas está muy condicionada por factores ambientales, principalmente los climáticos que, como es sabido, en montaña varían notablemente según la altitud. Sin embargo, a una escala más concreta, y como ya hemos ido indicando, la inclinación de los taludes y los procesos de erosión que en éllos se desarrollan parecen determinar el porcentaje de cubrimiento vegetal.

\section{Tabla 3. Cubrimiento medio de los distintos tipos de vegetación según la altitud (\%)}

\begin{tabular}{|l|c|c|c|c|c|c|}
\hline & $<800 \mathrm{~m}$ & $800-1200$ & $1200-1600$ & $>1600$ & Anova & sign. \\
\hline C. Arbóreo & 0.3 & 6.8 & 16.5 & 38.7 & 9.86 & 0.000 \\
C. Matorral & 16.5 & 9.1 & 23.1 & 11.4 & 5.98 & 0.000 \\
C. Herbáceo & 19.4 & 11.6 & 15.7 & 20.1 & 1.71 & 0.163 \\
Total & 36.2 & 27.5 & 55.3 & 70.2 & 12.97 & 0.000 \\
\hline
\end{tabular}

En la Tabla 3 se observa el recubrimiento vegetal de los taludes en relación con distintos grupos altitudinales. Los datos indican que el cubrimiento vegetal se incrementa a medida que ascedemos en altitud -condiciones climáticas más frescas y húmedas- pasando de un $36.2 \%$ por debajo de $105800 \mathrm{~m}$ al $70.2 \%$ por encima de los $1600 \mathrm{~m}$. Este incremento es muy notorio en la recolonización por parte de la vegetación arbórea, casi inexistente por debajo de los $800 \mathrm{~m}$ y muy representativa a partir de los $1200 \mathrm{~m}$.

La altitud no sólo determina el mayor o menor cubrimiento vegetal de los taludes, sino también las especies más representativas. De hecho éstas se van sucediendo según los distintos pisos bioclimáticos por donde discurre la pista 
forestal. Por encima de los $1600 \mathrm{~m}$ el cubrimiento arbóreo de los taludes está representado por el haya (Fagus silvatica) que da paso en los sectores más continentalizados al pino (Pinus silvestris) (P2). Allí donde aparece el matorral las especies dominantes son los brezos (Erica australis ssp. aragonensis) y la brecina (Calluna vulgaris) (P1 y P2). En los tramos de pistas forestal por encima de los $1800 \mathrm{~m}$ (P2) los taludes aparecen recolonizados por brezales de Erica aragonensis, brecina, enebrales rastreros Ouniperus nana), o piornos (Cytisus purgans).

Entre los 800-1600 m se encuentran la mayor parte de los trazados de las pistas forestales analizadas. En esta amplia franja altitudinal los árboles más representativos en los taludes son el quejigo (Quercus faginea), en suelos calizos y altitudes bajas, el roble (Quercus pyrenaica), sobre todo en suelos silíceos, y el haya (Fagus silvatica). De los tres tipos de bosque, dada su facilidad para brotar, el rebollo es el que repuebla con más facilidad los taludes. Con respecto al matorral, en las pistas más occidentales (P1 y P2) destacan las escobas (Genista florida), retama (Cytisus scoparius) y brezo blanco (Erica arborea), que a mayor altitud enlazan con las comunidades de Erica aragonensis. A medida que nos trasladamos hacia el este son otras las especies a reseñar. En suelos silíceos (P5, por ejemplo) predomina la jara (Cistus laurifolius) acompañada del cantueso (Lavandula stoechas), Thymus mastichina, Thymus zygis y varias rosáceas. Estas comunidades de naturaleza xerófila y heliófila poseen una gran capacidad de penetración, por lo que no es extraño verlas invadiendo no sólo los taludes, sino los bordes, e incluso el interior de la calzada de la pista forestal. En substrato calizo (P4) se encuentran una serie de matorrales basófilos encuadrados dentro del denominado aulagar montano, con especies como Genista bispanica (aulagino), Gesnita scorpius (aulaga), Lavandula latifolia (espliego), Thymus vulgaris (tomillo), Rosmarinus officinalis (romero). También en suelos básicos sobre calizas (P3) aparece el boj (Buxus sempervirens) acompañado del endrino (Prunus spinosa), rosal (Rosa canina y Rosa pouzinii), el majuelo (Crataegus monogyna) y Erica vagans.

El papel del factor pendiente puede observarse en la Tabla 4. Con un nivel de significación del $98 \%$, el porcentaje de cubierta vegetal va descendiendo notablemente a medida que la pendiente se incrementa, pasando de un $62.9 \%$ para taludes con menos de $30^{\circ}$ a un $32.8 \%$ para aquellos que tienen una inclinación de más de $50^{\circ}$. Es necesario mencionar que por especies el porcentaje de cubrimiento del arbolado es muy semejante en todas las clases de pendiente. El potente y profundo aparato radicular de los árboles es más ajeno a las dificultades que la pendiente puede presentar a la colonización de los taludes. 
REVEGETACIÓN NATURAL EN TALUDES DE PISTAS FORESTALES

Tabla 4. Cubrimiento medio de los distintos tipos de vegetación según la pendiente del talud (\%)

\begin{tabular}{|l|c|c|c|c|c|c|}
\hline & $<30^{\circ}$ & $30-40^{\circ}$ & $40-50^{\circ}$ & $>50^{\circ}$ & Anova & sign. \\
\hline C. Arbórea & 11.3 & 13.6 & 10.2 & 10.6 & 0.28 & 0.836 \\
C. Matorral & 32.7 & 13.8 & 10.0 & 13.4 & 5.36 & 0.001 \\
C. Herbácea & 18.9 & 12.2 & 17.4 & 8.8 & 2.33 & 0.073 \\
Total & 62.9 & 39.6 & 37.6 & 32.8 & 3.05 & 0.028 \\
\hline
\end{tabular}

Para conocer la relación existente entre los procesos de erosión y la recolonización vegetal se han estudiado el arroyamiento difuso severo, las incisiones y los deslizamientos. Calculada su importancia en los 118 taludes muestreados, se han establecido, por medio de un análisis estadístico (cluster), tres grupos o modelos de evolución geomorfológica de taludes (Fig. 4). Dentro de cada uno de estos grupos se ha estudiado el porcentaje de cubrimiento vegetal (Tabla 5).

Tabla 5. Cubrimiento medio de los distintos tipos de vegetación según los modelos geomorfológicos (\%)

\begin{tabular}{|c|c|c|c|c|c|}
\hline & Modelo I & Modelo II & Modelo III & Anova & niv. sign. \\
\hline Talud T1 & 27.82 & 12.63 & 9.94 & 3.10 & 0.052 \\
Talud T2 & 68.35 & 26.29 & 52.16 & 7.34 & 0.001 \\
\hline
\end{tabular}

Modelo I. Incluye el $57 \%$ de los casos y manifiesta una erosión moderada tanto en el talud superior (T1) como en el inferior (T2). Los porcentajes de erosión por deslizamiento y arroyamiento difuso severo son del $14.4 \%$ y $3.1 \%$ respectivamente en el primer caso y el $4.2 \%$ y $8.4 \%$ en el talud inferior. En este grupo de pistas es menos importante el cubrimiento vegetal del talud superior (27.82 \%) que el del inferior (68.35\%). Esto va a ser una constante en los tres modelos, ya que en algunos casos (modelo I y II) la actividad de los deslizamientos impide o retrasa el avance de la vegetación. En el modelo III es la actividad de la escorrentía superficial la que limita los procesos de recolonización vegetal del talud superior. 
V. LARREA SÁENZ - J. ARNÁEZ VADILLO - L. ORTIGOSA IZQUIERDO
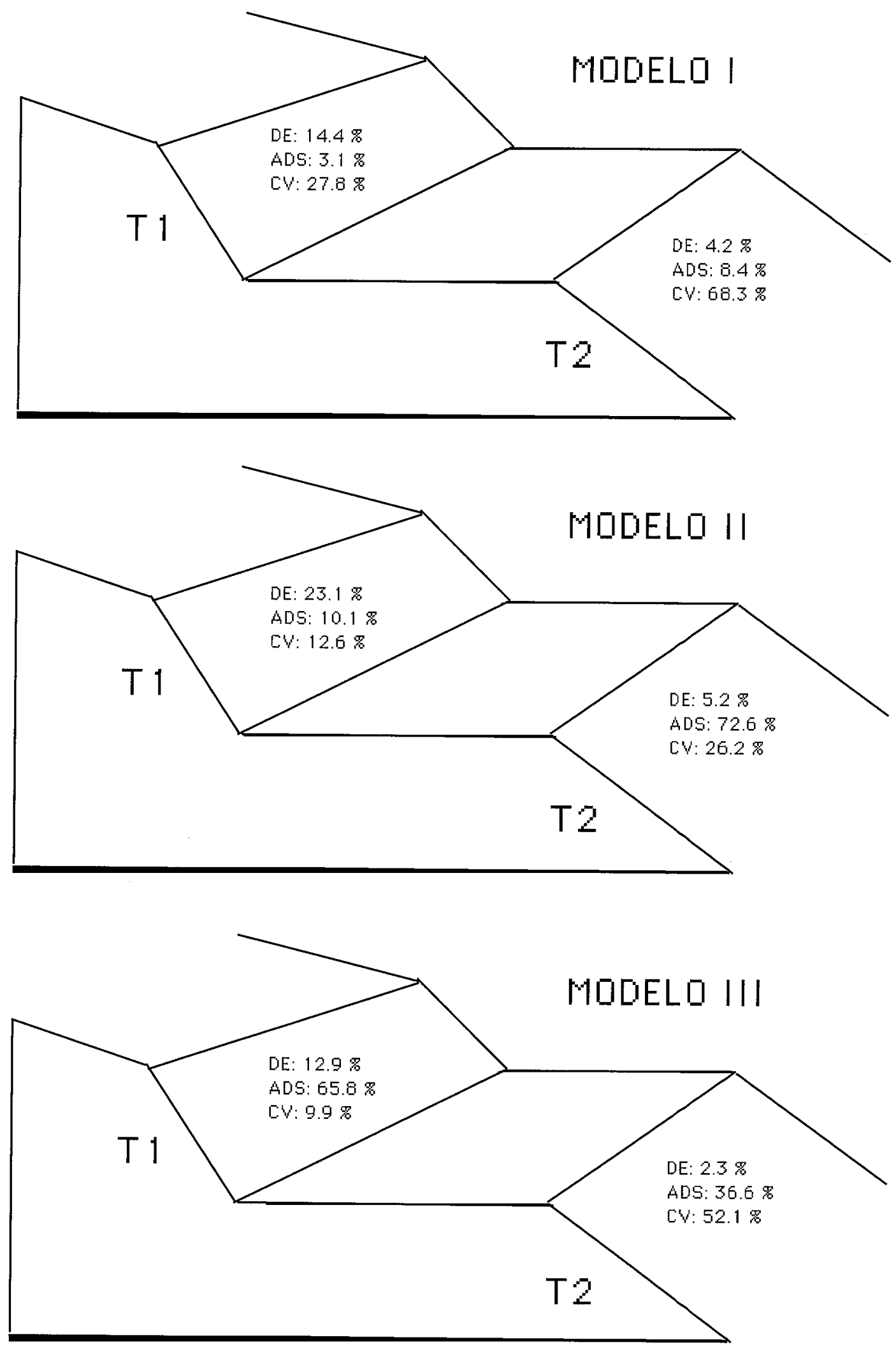

Figura 4. Modelos de erosión (con procesos más significativos) y cubierta vegetal. DE: deslizamientos; ADS: arroyamiento difuso severo; $C V$ : cubierta vegetal. 
Modelo II. Agrupa a 16 casos (27.1\%) y se caracteriza por el predominio del arroyamiento difuso severo en el talud inferior (T2), que se ve afectado en un $72.6 \%$ de su superficie. También destaca el papel de los deslizamientos en el talud superior (T1), con una ocupación del $23.1 \%$. Este modelo presenta importantes problemas de erosión, lo que repercute negativamente en el cubrimiento vegetal. La importancia del arroyamiento difuso severo en el talud inferior $(72.6$ \%) justifica el bajo porcentaje de cubrimiento vegetal: un 26.29\%. En ARNÁEZVADILLO \& LARREA (1994) ya se puso de manifiesto las relación que se establece entre las escorrentías superficiales y los taludes inferiores (T2) desprovistos de vegetación.

Modelo III. Incluye 9 casos en los que destaca el arroyamiento difuso severo e incisiones en el talud superior (T1), con un $65.8 \%$ del talud afectado. La importancia de este tipo de procesos queda reducida en el talud inferior (36.6\%). El cubrimiento vegetal, como es lógico, mejora en el talud inferior con respecto al modelo anterior (52.16\%) y empeora en el superior $(9.94 \%)$

\section{Conclusiones}

Los taludes de pistas forestales son sectores sometidos a diversos procesos de erosión. Para controlarlos se diseñan y aplican diversos sistemas de control entre los que se encuentra la restauración por medio de la vegetación. En los taludes sin medidas de protección una lenta recolonización vegetal, a partir de la vegetación existente en la ladera, entra en competencia con la erosión.

En las pistas estudiadas del Sistema Ibérico riojano se han observado diferencias significativas con relación al cubrimiento vegetal de los taludes. En concreto, el corte-talud superior (T1) presenta una revegetación más lenta y menos densa. La excesiva pendiente del talud y la actividad de los movimientos en masa (deslizamientos, coladas de barro, etc), muy activos en primavera como consecuencia de la saturación de los taludes, dificultan el enraizamiento y crecimiento de las plantas. El talud inferior (T2) -configurado por los derrubios procedentes del desmonte necesario para construir la pista- dispone de una mayor cubierta vegetal. Aquí la menor pendiente y las propias características del material sí favorecen el desarrollo vegetal. Es en estos taludes donde la vegetación se manifiesta como un eficaz control del arroyamiento difuso severo y de las incisiones. Mayores tasas de intercepción y evapotranspiración justifican un menor aporte de agua al suelo, pero además la vegetación se encarga de reducir la actividad del impacto de las gotas de lluvia y, en algunos casos, de retener la velocidad del agua que circula por el suelo.

El avance de la vetegación en los taludes de las pistas sigue unas pautas muy definidas. En el talud superior (T1) la vegetación penetra en forma de orla o banda desde la ladera situada aguas arriba de la pista, lo que se deduce por el 
mayor cubrimiento vegetal del tercio superior del talud. De la misma forma aparece otra orla al pie del talud vinculada a las buenas condiciones de humedad, acumulación de material suelto y recepción de semillas. En el talud inferior (T2) el proceso de la recolonización difiere con respecto a T1. Partiendo de la vegetación existente en la ladera, las plantas van avanzando desde el pie del talud, aunque no debe olvidarse que parte de esta vegetación -sobre todo la cubierta arbórea- puede ser prevía al vertido de los derrubios, habiendo permanecido parcialmente cubierta.

\section{Referencias bibliográficas}

ARNÁEZ-VADILLO, J. \& LARREA, V. (1991): Environmental and topographical controls in geomorphological evolution of hill-roads (Iberian System, La Rioja, Spain): In: Soil Erosion Studies in Spain (M. Sala, J.L. Rubio \& J.M. García-Ruiz, Edrs). Geoforma Ediciones, 27-39.

ARNÁEZ-VADILLO, J. \& LARREA, V. (1994): Erosion models and hydrogeomorphological functioning on hill-roads (Iberian System, La Rioja, Spain). $Z$. Geomorphologie, 38: 343-354.

ARNÁEZ-VADILLO, J. \& LARREA, V. (1995): Erosion processes and rates on roadsides of hill-roads (Iberian System, La Rioja, Spain). Pbysics and Chemistry of the Earth, 20 (3-4): 345-350.

DISEKER, E.G. \& RICHARDSON, E.C. (1962): Erosion rates and control methods on Highways cuts. Transactions of A.S.A.E., 5: 153-155

FOLCH GUILLÉN, R. (1977): El caso de las carreteras de montaña. En: Sobre Ecologismo y Ecología Aplicada. Ed. Ketres. Barcelona.

FOOKES, P.G.; SWEENWY, M.; MANBY, C.N.D. \& MARTIN, R.F. (1985): Geological and geotechnical engineering aspects lowcost roads in mountainous terrain. Eng. Geol., 21: 1-152

LARREA, V. \& ARNÁEZ VADILLO, J. (1996): Dinámica geomorfológica en pistas forestales de montaña: resultados en el Sistema Ibérico occidental (La Rioja, España). Zubía (en prensa).

NICOLAU IBARRA, J.M. (1992): Pauta estacional de la escorrentía en taludes de escombreras mineras en ambiente mediterráneo continental (Cuenca de Utrillas, Teruel). En: Estudios de Geomorfología en España (F. López Bermúdez, C. Conesa \& M.A. Romero Díaz, Edrs.) Sociedad Española de Geomorfología, pp 465-476.

NÚÑEZ OLIVERA, E. \& MARTÍNEZ ABAIGAR, J. (1991): El clima de La Rioja. Análisis de precipitaciones y temperaturas. Consejería de Agricultura. Gobierno de La Rioja. Logroño, 322 pag. 